\title{
Construcción de competencias requeridas en la investigación educativa y la sistematización de la docencia, según las percepciones de estudiantes de Educación Preescolar, Primaria y Secundaria de la Universidad de Costa Rica
}

\section{Competencies Required in Education Research and Teaching Systematization according to Students from the Preschool, Primary and Secondary Education Programs of the University of Costa Rica}

\author{
Mario Alberto Segura Castillo' \\ Facultad de Educación \\ Universidad de Costa Rica \\ San José, Costa Rica \\ mseguraster@gmail.com
}

Recibido 30 de mayo de 2012 - Corregido 08 de octubre de 2012 • Aceptado 07 de noviembre de 2012

\begin{abstract}
Resumen. El presente artículo tiene como finalidad presentar los resultados de dos foros virtuales, en los cuales participaron 42 estudiantes de la Licenciatura de Educación Preescolar, Licenciatura de Primaria y Licenciatura de Secundaria de la Universidad de Costa Rica. El objetivo principal de los foros fue determinar las percepciones que tienen los estudiantes participantes acerca de las competencias que han adquirido en el campo de la investigación educativa, además, cuáles han sido las herramientas fundamentales para poder sistematizar su propias prácticas de docencia. Los foros de discusión se llevaron a cabo en el curso de Métodos de Investigación Educativa (FD5091) de la Escuela de Formación Docente de esta Universidad, en los meses de marzo y abril del 2010. La muestra de estudiantes participantes estuvo constituida por $60 \%$ de estudiantes de Educación Preescolar, 35\% de Primaria y el 5\% de Educación Secundaria en las especialidades de Ciencias, Matemática y Estudios Sociales. Según las percepciones y creencias de las estudiantes participantes en los foros, tanto de los futuros docentes, como los que ya ejercen la profesión, a nivel de su preparación académica y en la situación actual de trabajo no cuentan con muchas posibilidades de investigar y sistematizar su propia mediación docente.
\end{abstract}

Palabras claves. Investigación, investigación educativa, competencias, sistematización, estudiantes, percepciones, formación universitaria.

Doctor en Educación con especialidad en Mediación Pedagógica de la Universidad La Salle, Costa Rica. Magíster en Evaluación Educativa, Universidad de Costa Rica; licenciado en Administración Educativa de la Universidad de Costa Rica. Magíster en Educación en Democracia y Valores en Iberoamérica, Universidad de Barcelona (2002-2003). Profesor universitario de la Universidad de Costa Rica y la Universidad Nacional. 
URL: http://www.una.ac.cr/educare

Abstract. The purpose of this paper is to present the results of two online forums carried out with the participation of 42 students of the Licenciaturas ${ }^{2}$ in Preschool Education, Primary Education and Secondary Education of the University of Costa Rica. The main purpose of the forums was to determine the insights of the participant students about the competencies they have achieved in the field of education research, and which have been the essential tools for them to systematize their own teaching practices. The discussion forums were part of the course FD5091 Métodos de Investigación Educativa [Education Research Methods] of the School of Teacher Education, delivered from March-April 2010. Of the sample, 60 percent were students of the Preschool teaching program, 35 percent were from the Primary Education teaching program and 5 percent were from the Secondary Education teaching program in the fields of Science, Mathematics and Social Studies. According to the insights and beliefs showed by the participants -both, the future teachers and the profession practitioners-, there are no opportunities for research or systematization of their own teaching mediation, in the current work situation.

Keywords. Research, Educational Research, Educational Research, Competencies, Systematization, Student Perceptions, university training.

\section{Acercamiento a la investigación educativa}

La investigación educativa es el proceso por el cual se encuentra la respuesta a los problemas que se le van presentado al personal docente en su quehacer cotidiano. Por medio de procesos investigativos se puede ir mejorando la calidad de la educación, por lo tanto, es de suma importancia considerar que en la formación de la población estudiantil en el campo de la investigación se puedan desarrollar competencias metacognitivas, que le permitan obtener conciencia acerca de su quehacer, para que asuma una posición creativa, crítica y transformadora y no simplemente repetitiva. Efectivamente:

En la investigación pedagógica el investigador es usualmente una persona comprometida con la educación (profesor, administrativo o especialista en educación) que quiere utilizar el enfoque cualitativo para hacer lo que considera más conveniente, bien sea para mejorar la enseñanza en términos generales o su propia enseñanza; o bien para reflejar el grado de eficacia que tiene en su tarea y cómo puede mejorarla (...). (Álvarez, 1986, p. 17)

La investigación en el ámbito educativo es uno de los temas más novedosos para ser analizados a nivel de formación inicial en las universidades formadoras de docentes. Es de suma importancia que se considere desde el diseño curricular de las carreras y desde el desarrollo profesional en cuanto a que se trate como una competencia transversal que debe estar presente en todos los cursos $y$, particularmente, en los cursos específicos de investigación educativa-sistematización.

2 Translator's Note: In Costa Rica, the "Licenciatura" is a one-year post-Bachelor study program, usually including thesis. "Primary Education" refers to students from the 1st to 6th grades, and "Secondary Education" refers to students from the 7th to 11 th grades. 
En el caso de la carrera de Bachillerato y Licenciatura en Educación Preescolar y Secundaria, específicamente en el plan de estudios de bachillerato, solamente hay un curso específico para investigación: "FD-0545 Investigación para el mejoramiento del aprendizaje", que en su planteamiento se desarrolla como un curso teórico-práctico y que abarca múltiples dimensiones esenciales de la investigación en general y pedagógica. El problema que se presenta es la falta de tiempo para el desarrollo de esas dimensiones en el ámbito investigativo, debido a que los aspectos prácticos (por ejemplo, realizar observaciones y entrevistas) quedan por fuera, según lo expresado por la población estudiantil en los dos foros.

En el tema de la Educación Primaria, a nivel de Bachillerato se recibe el curso anterior y además, el de "Investigación-Acción" que permite la resolución de problemas socioeducativos y concienciar a la población estudiantil acerca de los procesos de sistematización de su labor docente. El programa del curso FD-0545, Investigación para el mejoramiento del aprendizaje", lo reciben estudiantes tanto de Educación Primaria como de Secundaria, en el programa del curso impartido en el III ciclo del 2008, se describe como:

El curso posibilita al estudiante el conocimiento de las bases teóricas y metodológicas que orientan la investigación educativa. Incluye una introducción a los enfoques cualitativos, cuantitativos y mixtos, a la identificación de problemas de investigación en el aula, y al planeamiento y ejecución de un proyecto de investigación. El curso además promueve la adquisición de una actitud para el mejoramiento profesional mediante la investigación. La metodología del curso parte del diagnóstico inicial que el equipo de docentes aplica, para tomar en cuenta las necesidades de los estudiantes). (Universidad de Costa Rica, Facultad de Educación, Escuela de Formación Docente, 2008, p. 1)

Lo anterior, se contrapone a las percepciones de la población estudiantil, pues considera que los cursos de investigación son poco prácticos y muy teóricos. Algunas de las principales preocupaciones fueron estas:

Sumamente teóricos en donde el docente solamente explica lo que el estudiante debe realizar, dejando por fuera la orientación práctica que le permita al alumnado comprender las bases y herramientas necesarias para elaborar una adecuada investigación. (E.Código2.32, foro, 6 de abril de 2011)

Enfoque conductista y magistral, en donde el estudiantado termina el mismo sin adquirir conocimientos valiosos, evitando de esta forma la realización en un futuro de su propia investigación. (E.Cod.2.25 foro, 6 de abril de 2011)

En la Universidad nos dan las herramientas para ejercer esta actividad, pero es nuestro compromiso como futuros educadores el realizarla con las condiciones adecuadas y con el propósito de mejorar la calidad de la educación. (E.cod2.22, foro, 6 de abril de 2011) 
Lo anterior no quiere decir que el estudiantado termine siendo el mismo, sin haber construido nuevos conocimientos, lo que sucede es que muchos estudiantes no logran concretar la teoría estudiada y ello les limita el aprendizaje, por lo que es importante que los cursos cumplan el requisito de ser teórico-prácticos, pues solo se aprende a investigar, investigando. (E.cod2.13, foro, 6 de abril de 2011)

Dentro de las percepciones en ambos foros, la población estudiantil considera que, en el ejercicio profesional, el personal docente asume muchos papeles que no se limitan a simplemente impartir las lecciones. Por ejemplo, deben funcionar como madres, padres, enfermeras, psicólogos y psicólogas y otros. Además, consideran que se requiere la disposición hacia el estudiantado, con el fin de brindarles los recursos necesarios para formarse como seres integrales.

Por ende, ser propulsores del mejoramiento de las condiciones del medio escolar donde se labora y una herramienta importante para cumplir tal fin, requiere de la investigación educativa y la sistematización.

En torno a lo anterior, la importancia de la investigación educativa, según opiniones de la población estudiantil, es poder convertirla en una importante herramienta dentro del proceso educativo. A través de esta, el personal docente tiene la oportunidad de reflexionar en torno a su propia práctica, reconocer los problemas que presenta el grupo o grupos que donde se lleva a cabo el quehacer pedagógico y, de esta forma, innovar; es decir, buscar soluciones sencillas y específicas, evidentemente coherentes con la realidad que se esté viviendo, en pro de mejorar su propia práctica profesional y, en consecuencia, contribuir a generar un sistema educativo de calidad.

En las discusiones formuladas en los foros (2 de abril del 2010), analizadas por mención de respuesta, se considera la investigación educativa como un proceso fundamental de la profesión docente, debido a que cada realidad de aula es diferente, por ejemplo, cada estudiante aprende a su propio ritmo y con diferente estilo de aprendizaje, la situación económica de cada contexto es particular, así como la familia, cada grupo y estudiante son particulares y los procesos que se construyen cada día son únicos e irrepetibles. Al respecto opina:

No obstante, la investigación educativa como factor importante en el desempeño pedagógico debería ofrecer oportunidades para ser sistematizada, en especial cuando esta resulta ser extensiva. Es ahí donde entra la importancia y la posibilidad de que los docentes busquen capacitarse y hacer públicos sus hallazgos por sus propios medios, simplemente porque les interesa y no solo porque se les impone. (E.Cod2.1. 2 de abril, 2010)

De acuerdo con dicha opinión y según Céspedes y Castro (2010), el sistema educativo de la educación formal en Costa Rica, actualmente, no ofrece las condiciones adecuadas para llevar a cabo procesos investigativos, máxime, por la falta de tiempo para la recogida de la información y la sistematización que alegan tener el personal docente y administrativo. 
Igualmente, existe poca preparación en la formación inicial para que el personal docente se empodere de los procesos de investigación y sistematización dentro de su quehacer.

Asimismo, Céspedes y Castro (2010) indican que los procesos de capacitación en investigación dirigidos al personal docente son casi nulos. Pero también, existe mucho temor en el cuerpo docente a realizar investigación, debido a que la elaboración de tesis ha constituido un proceso arduo. Sin duda, cuando el personal docente se concientice de investigar su propia práctica profesional y sistematizarla, podría causar un impacto positivo en el aula, como un factor determinante que mejora sus procesos de enseñanza y aprendizaje.

La investigación del aula le permite al personal docente romper esos grandes mitos que existen respecto de los procesos investigativos. No se requieren grandes recursos para llevar a cabo procesos de sistematización de la práctica cotidiana, que le permitan la reflexión y discusión para el mejoramiento continuo del quehacer.

En cuanto a la sistematización, según Kisnerman y Mustieles (1997), es “(...) el procedimiento y conjunto de operaciones que ordena, describe, articula y recupera el desarrollo de una experiencia práctica, conectando los datos empíricos que en ellas se obtienen con una determinada teoría" (p. 13).

De acuerdo con lo anterior, el estudiantado participante en los foros considera que no hay tiempo para realizar procesos de investigación y luego de sistematización de su quehacer, debido a la gran carga administrativa que día tras día tienen que cumplir, lo que le limita a no poder investigar para luego sistematizar su práctica.

Un docente que no sistematice, es un docente inerte, realmente se debe de promover la investigación dentro de la propia aula, porque:

Una vez acontecida la práctica, la sistematización es un proceso participativo que permite ordenar lo acontecido, recuperar así la memoria histórica (es todo aquel acontecimiento, hecho significativo, procesual y asumido a través de la realidad social), interpretarla, aprender nuevos conocimientos y compartirlos con otras personas. Por lo que las etapas, pudieran ser:

- Un proceso en construcción en el que hay un itinerario-lógica-ordenamiento.

- Incluye un proceso-flujograma-secuencia, como el producto-evidencia.

- Es un sumario abierto a las aportaciones y el desarrollo del grupo.

- Ayuda a descubrir la lógica (realidad) con la que ese proceso, la actividad que se realiza, que se llevó a cabo (factores, relaciones, etc.). (Anguiano et al., 2009, párr. 7-11) 
URL: http://www.una.ac.cr/educare

Una de las competencias requeridas por los docentes en la actualidad es su capacidad de sistematizar, igualmente, de actuar para el mejoramiento de los centros educativos.

\section{Aspectos metodológicos del foro}

Con la finalidad de presentar los resultados de los dos foros donde participaron 42 estudiantes de Educación Preescolar, Primaria y Educación Secundaria de la Universidad de Costa Rica (UCR), que actualmente cursan la Licenciatura en la Escuela de Formación Docente, se realizaron dos foros virtuales de discusión para determinar las competencias que requieren los docentes en el campo de la investigación. La población estudiantil manifiesta su inconformidad con su formación en el campo de la investigación educativa, debido a que, según su opinión, los cursos de dicho componente no trascienden al plano vivencial por su carácter eminentemente teórico. También, se hizo necesario conocer las competencias que consideran fundamentales en dicho campo.

Los foros de discusión se llevaron a cabo en el curso de Métodos de Investigación Educativa de la Escuela de Formación Docente de esta Universidad, durante el I Semestre del año 2010. Los foros fueron de forma virtual, para el primero se creó un correo electrónico, las percepciones de la población estudiantil de dicho foro se sistematizaron por el docente del curso y luego se creó un "BLOGS" para comentar la síntesis del primer foro. Los foros se realizaron del 16 de marzo al 6 de abril del 2010 (Segura, 2010).

A partir de lo anterior, surge la idea de determinar las percepciones acerca de los procesos de formación que ha recibido la población estudiantil en el campo de la investigación educativa, así como conocer las competencias que consideran fundamentales.

Desde un punto de vista metodológico, la presente investigación se fundamenta en la perspectiva cualitativa, debido a que el estudiantado participante en los foros manifiesta los valores, las percepciones y los intereses de su proceso de formación en el tema de la investigación educativa; asimismo, se convierte en un proceso holístico, multicausal y complejo, debido a que las percepciones del estudiantado se relacionan con procesos de investigación, sistematización y elaboración de competencias adquiridas durante su proceso de formación docente.

La intervención en los foros se realizó mediante un mínimo de dos participaciones por estudiante, a partir de la lectura previa del artículo de Céspedes y Castro (2010), El docente como investigador de su quehacer en Costa Rica. La discusión se centró en los aspectos relativos al campo de la investigación, sistematización y competencias de dicha investigación.

Dentro de las principales conclusiones, se visualiza que:

- (...) Estos profesionales se ven a símismos como carentes de formación en investigación y ubican la investigación como la primera de las áreas por fortalecer en la formación inicial de docentes. 
- Los docentes y los graduados no asocian la investigación con las debilidades formativas en otras áreas del quehacer docente ni la ubican como herramienta coadyuvante de la mejora de los procesos educativos. (...)

- Los participantes de la investigación no manifiestan poseer alto dominio de competencias investigativas, pese a que consideran que el quehacer investigativo es un factor importante para el mejoramiento de la calidad educativa y para la innovación. Los participantes reconocen la importancia de fortalecer su formación en el campo investigativo y la necesidad de realizar cambios en las condiciones laborales del docente, para que les permitan una práctica profesional en donde las decisiones pedagógicas se asienten en una base científica y en propuestas pertinentes para los contextos educativos específicos en los que se desempeñan. (Céspedes y Castro, 2010, p. 20)

Aunado a ello, el docente del curso de Métodos y Técnicas de Investigación construye las categorías de análisis emergentes, las cuales se sistematizan para llegar a conclusiones por mención de respuesta:

a. Competencias adquiridas en los cursos de investigación educativa

b. El proceso de sistematización e investigación

c. Percepciones de los cursos de investigación

d. Iniciar el proceso de investigación educativa-sistematización

La sistematización de la participación del estudiantado en los foros se hizo a partir de las siguientes preguntas generadoras: ¿Cuáles son las percepciones que tienen de los cursos de investigación educativa? ¿Cuáles son las competencias que adquirieron como investigadores e investigadoras en los cursos de investigación educativa durante su formación inicial? ¿Cómo iniciar el proceso de investigación educativa?

Por su parte, los procesos de triangulación para comprobar la fiabilidad de la información de los foros, están basados en los foros como grupo de discusión y en el análisis de contenido como técnica hermenéutica que permite la interpretación de los aportes de las personas que participaron y por mención de respuesta de las personas participantes.

A continuación se presenta, según la opinión de las personas participantes en los dos foros, cómo inician el proceso de investigación-sistematización. 


\section{Formas de iniciar los procesos de investigación educativa}

Las personas participantes en el foro consideran que los cursos de investigación en el Bachillerato de la Educación Preescolar, Primaria y Secundaria proporcionan las bases teóricas, pero no prácticas para que los futuros profesionales adquieran competencias básicas para lograr sistematizar su propio quehacer.

Es importante que a nivel de los cursos de investigación educativa sea necesaria la transversalización de herramientas que se vinculen directamente con la investigación y la sistematización. Asimismo, según las estudiantes y los estudiantes participantes en los foros, el cuerpo docente tiene diferentes posiciones acerca de cómo se elabora la investigación en ambos paradigmas, por lo que es necesario la unificación de criterios a nivel de formadores. La investigación educativa requiere, en todas las asignaturas del plan de estudio en las carreras de educación de la UCR, un lenguaje común para poder desarrollar las competencias que, según las percepciones del estudiantado participante de los foros (marzo-abril 2010), hace falta promover, como son la escucha, el trabajo en equipo, la problematización de la realidad, entre otras.

La población estudiantil manifiesta que, en los cursos, muchas veces solo se hace la práctica de las secciones que requieren un informe final de investigación y el personal docente se contradice acerca de cómo se formulan, ahí es donde se produce la confusión.

Asimismo, según sus percepciones, en el quehacer cotidiano del personal docente desde la Educación Preescolar hasta la Educación Secundaria, los problemas que se pueden investigar se detectan o ya se conocen; sin embargo, es muy difícil darles seguimiento, ya sea por su elevado costo económico, el trabajo extra o por los intereses de los entes involucrados. Esto hace que investigar se convierta en un simple requisito de un curso a nivel universitario, pero cuando se ejerce la profesión tanto la investigación educativa como la sistematización quedan de lado del propio quehacer docente.

Por lo anterior, consideran que muchas veces sería más productiva una buena investigación con muchas personas involucradas, donde se aprenda en conjunto cada uno de los pasos, se lleguen a consensos a nivel del profesorado de la Escuela de Formación Docente y el estudiantado brinde un aporte, pequeño, pero sustancial, en el momento de la ejecución. Esta práctica sería más enriquecedora que una tesis de grado, las cuales, en la mayoría de los casos, se convierten en investigaciones olvidadas en las bibliotecas y de las que tampoco se aprende mucho, en los cursos de formación docente.

Además, la investigación-sistematización no son consideradas como labores inherentes a la docencia, sino más bien actividades de especialistas en el campo educativo de la ciencia y tecnología, por lo que desde la capacitación y formación permanente en docencia desde Educación Preescolar hasta Educación Secundaria, se hace necesario crear una cultura de sistematizar el quehacer pedagógico. Con una formación en las distintas carreras de educación de la UCR y la capacitación pertinente, los educadores lograrían aprovechar mejor las 
oportunidades de crear herramientas para investigar y sistematizar la práctica pedagógica y, a partir de la teoría existente, proponer estrategias innovadoras en su área.

La pregunta que surge es cómo iniciar los procesos de investigación educativasistematización en todos los niveles y ciclos del Sistema Educativo Costarricense y en qué consistiría la ayuda necesaria para sistematizar los procesos de mediación pedagógica que requieren los educadores: "Lo primero es el trabajo en equipo, saber comunicar lo que sucede en los procesos de mediación pedagógica, ser creativo y ser humilde cuando se sistematiza, Lo importante es aprender y mejorar, es decir, se busca perfeccionar el propio quehacer pedagógico, cuando se investiga o sistematiza" (Est.cod.2.10. foro. 2 de abril 2010).

Igualmente, para desarrollar los propios procesos de investigación, es necesario que el personal docente que quiera investigar su quehacer pedagógico, tenga claro que no solamente debe saber sobre pedagogía, sino que, además, debe tener la iniciativa y entusiasmo necesarios para utilizar herramientas que le permitan investigar su propia realidad y luego sistematizarla, tomando en consideración diversos aspectos o situaciones que causan incertidumbre o para los que no se tiene ningún tipo de respuesta para su solución inmediata.

Otro camino para iniciar el proceso de investigación para luego sistematizarlo en el aula es recordar que, para realizar una investigación educativa, es de suma importancia empezar a formular cuestionamientos o hipótesis acerca de la mediación pedagógica que se realiza cotidianamente, para comenzar a reflexionar sobre cuáles aspectos requieren una mejora inmediata y, de acuerdo con ello, ir poco a poco desarrollando diferentes estrategias didácticas para sistematizar los principales resultados y, consecuentemente, ir aplicando los pasos de la investigación. Por su parte, es importante comunicar los resultados de las sistematizaciones que se vayan realizando $y$, en caso necesario, solicitarles a otros profesionales ayuda en el campo de la investigación educativa-sistematización.

Cuando el cuerpo docente se capacite en el conocimiento de diferentes técnicas, métodos de investigación, construcción de información y sistematización de las experiencias, se permitirá aplicar las que sean más adecuadas en el contexto del centro educativo y, por lo tanto, de sus estudiantes, conjuntamente con la reflexión sobre la ética de su quehacer como una persona investigadora.

Las experiencias, en tanto procesos dialécticos, permiten tomar en consideración perspectivas objetivas y subjetivas en un contexto histórico social particular, por lo que se consideran de suma importancia las particularidades del contexto, donde la persona investigadora se encuentra inmersa. Por ejemplo, las situaciones específicas del centro educativo, nivel escolar y de la sección son únicas e irrepetibles, por lo tanto:

c) Una experiencia siempre está constituida por acciones; es decir, por cosas que hacemos (o dejamos de hacer) las personas. De forma intencionada o inintencionada; planificada o imprevista; dándonos cuenta de su realización o sin reconocerla mientras las realizamos. 
URL: http://www.una.ac.cr/educare

d) Pero también en toda experiencia se manifiestan las percepciones, sensaciones, emociones e interpretaciones de cada una de las personas que viven esas experiencias, es decir, de los hombres y mujeres protagonistas de las mismas.

e) Además, las experiencias incluyen, al realizarse, determinados resultados o efectos que modifican en todo o en parte los elementos o factores existentes anteriormente.

f) La interrelación de todos estos factores generan reacciones en las personas que intervienen, las cuales construyen relaciones entre ellas. Estas relaciones personales y sociales -por una parte- han sido mediadas por todos los elementos anteriores y -por otra- son factores desencadenados por lo que aconteció durante la experiencia. (Ayllón, 2002, p. 19)

De acuerdo con lo anterior, Sandoval (2001) considera necesario, para sistematizar una experiencia, partir de un proceso de contextualización de la realidad; luego, paulatinamente, proponer un orden lógico de dicha realidad y un discurso descriptivo de los acontecimientos mediante la interpretación crítica de lo que va aconteciendo en los procesos de mediación pedagógica, para lograr extraer conclusiones acerca del porqué suceden los acontecimientos.

Mediante el análisis y la reflexión, se antepone la teoría que fundamenta el quehacer y los resultados de la práctica pedagógica que permitan solucionar los problemas que investigamos. Tomando las mismas palabras de Sandoval (2001), el proceso de sistematización se refiere a:

(...) una interpretación crítica de la práctica que, a partir de su ordenamiento y reconstrucción, descubre o explicita la lógica del proceso vivido, los factores que han intervenido en dicho proceso, cómo se han relacionado entre sí, y por qué lo han hecho (...). (p. 116)

\section{Las competencias que permiten ser una persona investigadora y sistematizadora}

Una competencia profesional es un conjunto de habilidades, destrezas, conocimientos y valores que dinamizan un aprendizaje, el cual permite enfrentar situaciones durante toda la vida, por lo que para desarrollar procesos de investigación, el personal docente debe tener una actitud crítica, de compromiso con la realidad que le rodea, innovadora y activa para que sea capaz de proponer soluciones, cambios y mejoras. Braslavsky, Acosta y Jabif $(2004$, p. 42) consideran que "la competencia siempre remite a una construcción influida por el contexto, es decir: se actúa con una finalidad en un contexto específico en el que se combinan diferentes aspectos". 
Con base en lo anterior, el cuerpo docente debe contar con una formación adecuada en los procesos de sistematización e investigación áulica, las técnicas de construcción de información, que pueda analizarlas, y plantear posibles intervenciones y acciones que contrarresten las necesidades que encuentre en el contexto por investigar. Al respecto, una estudiante participante de los foros indicó que entre las principales competencias que requieren los docentes se encuentran:

- Capacidad para trabajar en equipo: es importante que los docentes aprendan a trabajar en conjunto, con grupos de docentes o con otras personas involucradas en el proceso de sistematización de experiencias, ya que no todas las investigaciones educativas se llevan a cabo individualmente, en su mayoría son grupales y exigen el acuerdo mutuo entre todas las partes con el fin de lograr una investigación de calidad que responda a todas las necesidades o problemas planteados al inicio de la investigación.

- Capacidad de escucha: durante la realización de una investigación educativa, se presentan problemas que muchas veces hacen flaquear al personal docente y dejar de lado dicha investigación, o, por otro lado, no son capaces de poder escuchar a determinadas personas que tratan de ayudar en el proceso de investigación y por este motivo falla o no se adapta a la problemática del lugar. Por esta razón, es fundamental que se escuchen los consejos o recomendaciones que nos pueden brindar desde docentes hasta los mismos padres de familia (que también son parte esencial de toda investigación educativa) y alumnado con el fin de retroalimentar la investigación y sobretodo tomar en cuenta a las personas que forman parte activa de los procesos de enseñanza y aprendizaje.

- Capacidad para problematizar la realidad educativa: esto más que todo orientado a desarrollar la capacidad de contextualizacion basada en la observación directa de los fenómenos que afectan a la población meta.

- Capacidad referencial: no se trata tan solo de saber o conocer sobre teorías que apoyen la práctica educativa o investigaciones de carácter menos formal, sino también de asociar las experiencias vividas y las estrategias implementadas en una situación particular a situaciones análogas, o bien, poder adaptarlas a la situación de interés.

- Capacidad vinculativa y de procesamiento de datos: un investigador-sistematizador, debe ser capaz de unir teorías, reunir datos, y más que todo procesarlos de forma que sean legibles y entendibles al común denominador, que sean accesibles, porque ¿de qué sirve un excelente estudio cuyo vocabulario sea tan elevado que tan solo los "eruditos" del tema sean capaces de comprenderlo, cuando su aplicación involucra a todos los sectores?

- La habilidad de ser un buen observador. 
URL: http://www.una.ac.cr/educare

- Habilidad para poder analizar diferentes fuentes de información.

- Capacidad para analizar la realidad educativa costarricense.

- Conocimiento del desarrollo de procesos de investigación.

- Constante actualización en materia de software y aprovechamiento de las tecnologías de la información y comunicación. (Est.Cod.1. 25 Foro 16 de Marzo del 2010)

Las anteriores competencias se refieren al trabajo en equipo, a la complejidad, de la capacidad de escucha, de ser una persona sistematizadora de datos y otras que tendrían que tomar en consideración en los cursos de investigación. Además, ponen en evidencia que en dichos cursos se requiere solventar los problemas que día tras día se presentan en nuestra mediación, pero principalmente poseer compromiso, es decir, el docente debe estar dispuesto a ir más allá del contexto del aula, dedicándose a analizar a fondo las necesidades de la población estudiantil y los contextos en los que está inmersa; para ello, debe busca capacitarse y reflexionar sobre las diferentes vías de acción. De nada sirve conocer en detalle cómo desarrollar una investigación, si no existe el compromiso para llevar a cabo este proceso de sistematización, el cual, si bien puede resultar extenuante, busca el fin de mejorar la práctica educativa.

Dicha mejora incluye entender como fundamentales la divulgación y la retroalimentación en la investigación como tal, ya que al comunicar los resultados, las técnicas utilizadas pueden responder a necesidades de otras comunidades y contextos educacionales, y generar modificaciones en el trabajo de investigación original, con miras al mejoramiento y al planteamiento de soluciones efectivas no solo a nivel macro sino micro, lo cual compete en mayor medida al docente como persona. En ese sentido, una estudiante participante de los foros indicó otra competencia fundamental:

Las investigaciones lucen bien en el papel, pero se les saca mayor provecho si se ponen en práctica. Por ejemplo, de nada nos sirve haber investigado sobre los métodos de lectoescritura en el nivel inicial, si no se toma el tiempo para analizar cuál es el mejor método para mi grupo o cómo puedo optimizar el proceso con cada uno de mis alumnos. Considero que un docente investigador, debe asumir el proceso completo, identificar el problema, buscar la solución y documentar por escrito lo investigado. Así se podrá compartir la información tanto para ayudar a otros como para que los demás también enriquezcan nuestro trabajo. (Est. Cod2. 20. 2011, foro, 5 de abril)

Con base en lo anterior, es de suma importancia que se conozcan las competencias fundamentales que requiere la población estudiantil que se forma en las diversas carreras de la Facultad de Educación de la Universidad de Costa Rica, para que les permita construir 
conocimientos, valores, habilidades y destrezas, mediante los procesos de investigaciónsistematización de su propia mediación pedagógica. La problematización, el trabajo en equipo, el diálogo y la actualización son competencias que actualmente requieren las personas que se forman en la investigación educativa.

\section{Consideraciones finales}

Según lo investigado por Céspedes y Castro (2010), con pocas asignaturas dentro de los diferentes planes de estudios de las carreras formadoras de docentes de las universidades públicas y privadas de Costa Rica, se le dificulta a la población estudiantil, adquirir los instrumentos teóricos/prácticos de una investigación, ya que esta requiere mayor tiempo por su complejidad. Por tanto, sería conveniente que en la mayoría de asignaturas se investigue y se brinden las herramientas necesarias, para lo cual se tendrían que correlacionar los programas de curso con la investigación educativa-sistematización.

En el sistema educativo, en general, existen múltiples temáticas por investigar y, en consecuencia, sería posible adquirir, mediante un proceso adecuado, las competencias necesarias para una formación en investigación y sistematización. Sin embargo, sin las herramientas básicas para investigar y sistematizar, dicha labor se convierte en una práctica poco usual en el Sistema Educativo Costarricense.

Por eso, todas las oportunidades existentes en el aula, la comunidad, en las interacciones entre las entidades escolares y entre profesionales son ignoradas para realizar procesos de investigación-sistematización. La calidad educativa también debería ser parte de procesos de sistematización que le permitan al personal docente adquirir competencias de trabajo en equipo, de escucha, de problematización y muchas otras de igual importancia.

Desde esta perspectiva, el personal docente como náufrago con un solo salvavidas y a la merced de una tormenta, no tiene más que aceptar sus limitaciones, por lo que el Ministerio de Educación Pública junto con las universidades, podrían iniciar procesos de asesoramiento y capacitaciones en la implementación de la investigación-sistematización como parte fundamental de la labor docente.

En síntesis, las percepciones y creencias de la población estudiantil participante en los foros se basa en que la situación actual, tanto a nivel de preparación académica de las personas que se forman para docentes, como los que ya ejercen la profesión, no cuentan con muchas posibilidades para lograr la capacitación real en cuanto al ámbito investigativo-sistematización.

Las personas que desde el aula investigan o sistematizan su experiencia la realizan por convicción personal, tampoco se cuenta con la cultura investigativa en el país para insertar la investigación-sistematización, desde su papel preponderante en la labor de cualquier profesional. 
URL: http://www.una.ac.cr/educare

La investigación educativa es una actitud que debe ser parte de los que eligen como profesión la educación. Si realmente el personal docente busca una mejor educación, es de suma importancia que se establezcan acciones, que le ayuden a generar cambios desde sus propios contextos. Una de estas acciones es la investigación educativa, junto a la constante y pertinente actualización. Al respecto, una participante de los foros (Est. Cod2. 27. Foro del 5 de abril, 2010) mencionó:

La escuela como una de las principales instituciones encargadas de la enseñanza de los niños y niñas de nuestro país, cada día se ve más envuelta por una nube de problemas, inconvenientes y deficiencias producto de las nuevas tecnologías, que poco a poco la van desplazando convirtiéndola casi por completo en un lugar obsoleto, en donde no se llevan acabo estrategias y técnicas acordes con el desarrollo de los niños y niñas o su estilo de vida, que le permitan a los estudiantes adaptarse a la sociedad que constantemente esta sufriendo cambios. Por esta razón es que como profesionales en el campo educativo, debemos informarnos, investigar y crear nuevas herramientas, logrando que los niños y niñas de nuestras aulas adquieran el conocimiento de una manera más activa y participativa, no solo por el hecho de hacer [sic] un ambiente ameno en donde todos cooperen y participen que también es muy importante, sino porque los niños (as) son más competitivos y lo exigen, ya que su entorno es muy distinto al que nosotras (os) vivimos cuando cursábamos nuestros estudios preescolares y escolares.

En la actualidad, se dice que la educación está en crisis, que se debe mejorar la calidad y desempeño del sistema educativo. Una forma muy efectiva es por medio de la implementación de la investigación educativa-sistematización que viene a enriquecer el quehacer educativo.

De acuerdo con Gelb (1999), los siete principios davincianos que deben acompañar al personal docente en su proceso cotidiano de investigación educativa son:

- Curiosità. La actitud de acercarse a la vida con una curiosidad insaciable y la búsqueda continua del aprendizaje.

- Dimostrazione. El compromiso de poner a prueba el conocimiento a través de la experiencia, la persistencia y la disposición a aprender de nuestros errores.

- Sensazione. El continuo refinamiento de los sentidos, especialmente de la vista, como medio para animar la experiencia.

- Sfumato (...). La voluntad de aceptar la ambigüedad, la paradoja y la incertidumbre. (...) 
- Corporalita. El cultivo de la gracia, la ambidestreza, la condición física y el porte.

- Connessione. El reconocimiento de la interconexión de todas las cosas y de todos los fenómenos. Pensar en términos de sistemas. (p. 10)

El motor de todo proceso de investigación y sistematización pedagógica es la sed por construir el conocimiento, de aprehender nuestros problemas cotidianos que dificultan que nuestro estudiantado llegue aprender a indagar y a solucionar las propias barreras de aprendizaje y, sobre todo, a transformar la realidad, para que lo fundamental en la formación inicial del profesorado sea entender la investigación desde lo transversal, lo complejo y lo imprescindible.

\section{Referencias}

Álvarez, J. M. (1986). Introducción a la edición española: Investigación cuantitativa/investigación cualitativa: ¿Una falsa disyuntiva? En T. D. Cook y C. S. Reichardt (Eds.), Métodos cualitativos y cuantitativos en investigación evaluativa (pp. 9-24). España: Morata.

Anguiano, A. M., López, S., Plascencia C., Jiménez, S., Perea, M. B. y Calvo, A. L. (verano, 2009). Reflexionar sobre la sistematización; una preocupación del profesional de Trabajo Social. Margen, 52. http://www.margen.org/suscri/margen52/anguian.html

Ayllón, M. R. (2002). Una propuesta operativa para sistematizar. Aprendiendo desde la práctica. Lima: Asociación Kallpa.

Braslavsky C., Acosta, F. y Jabif, L. (2004). Orientaciones conceptuales y didácticas. Buenos Aires: IIPE-UNESCO.

Céspedes, J. y Castro, D. (junio, 2010). El docente como investigador de su quehacer en Costa Rica. Patria Grande. Revista Centroamericana de Educación, 1(1), 1-23. Recuperado de http://www.estrategiaol.com/revista/index.php/patria grande/article/viewFile/1/4

Gelb, M. J. (1999). Inteligencia genial. 7 principios claves para desarrollar la inteligencia, inspirados en la ida y obra de Leonardo da Vinci. Bogotá: Norma.

Kisnerman, N., y Mustieles D. (1997). Sistematización de la práctica con grupos. Buenos Aires: Lumen Humanitas.

Sandoval, A. (2001). Propuesta metodológica para sistematizar la práctica profesional del trabajo social. Buenos Aires: Espacio Editorial.

Segura, M. (16 de marzo-6 de abril de 2010). Re: Acerca de las oportunidades que se tiene en el sistema educativo de realizar un proceso de investigación que permita formarme con competencias en dicho campo. [Foro en línea] Curso FD5091- Métodos y Técnicas 
de Investigación Educativa. Escuela de Formación Docente. Universidad de Costa Rica. Recuperado de <metodosfd5091.Blogspot.com> y <http: metodosfd5091@gmail.com>

Universidad de Costa Rica, Facultad de Educación, Escuela de Formación Docente (2008). FD-0545-Investigación para el mejoramiento del aprendizaje (Programa del curso). San José: Autor.

\section{Cómo citar este artículo, según APA:}

Segura, M. (2012). Construcción de competencias requeridas en la investigación educativa y la sistematización de la docencia, según las percepciones de estudiantes de Educación Preescolar, Primaria y Secundaria de la Universidad de Costa Rica Revista Electrónica Educare, 16(3), 77-92. Consultado de http://www.revistas.una.ac.cr/index.php/EDUCARE/issue/current

Nota: Para citar este artículo en otros sistemas puede consultar el hipervínculo "Como citar el artículo" en la barra derecha de nuestro sitio web:

http://www.revistas.una.ac.cr/index.php/EDUCARE/index 